\title{
Developments of power system protection and control
}

\author{
Z. Q. Bo ${ }^{1 *}$, X. N. Lin' ${ }^{2}$, Q. P. Wang ${ }^{1}$, Y. H. Yi ${ }^{1}$ and F. Q. Zhou ${ }^{1}$
}

\begin{abstract}
Synchronized wide area communication has become a mature technology, which makes the real-time interaction between the substations and the wide area protection and control system possible. However, the present protection and control system to handle this real-time data has been recognized to be deficient. This paper begins by reviewing the development history of power system protection, with special attention paid to the recent development in the field of wide-area and integrated protections, in order to look into the future development of protection and control systems. Then the concept of integrated wide area protection and control is introduced, where it can be shown that a hierarchical protection and control system provides the protection and control for wide area or regional power substations/plants and their associated power networks. The system is mainly divided into three levels: the local, the substation/plant, and the wide area/regional. The integrated functions at each level are described in details with an aim to develop an optimal coordination mechanism between each level. The key element in the proposed system is the wide area real-time protection and control information platform, which not only enables the merger of three lines of defence for power system protection and control, but also provides a perfect tool for the application of cloud computing in substations and power networks.
\end{abstract}

Keywords: Power system protection, Wide area protection, Integrated protection and control, Information platform

\section{Introduction}

Power system protection emerged at the beginning of the last century, with the application of the first electro-mechanical overcurrent relay. The majority of the protection principles currently employed in protection relays were developed within the first three decades of the last century, such as overcurrent, directional, distance and differential protection, as shown in Fig. 1. The development of modern science and technology, especially electronic and computer technology, promoted the development of relay technology, such as materials, components and the manufacturing process of the hardware structure of relay protection device. At the same time, great theoretical progress had been made in the relay protection software, algorithms, etc. As shown in Fig. 1, the progress in modern technology stimulates the development in power system protection. In the last century from the emergence of protection to the end of the 1990s, the relay protection had gone

\footnotetext{
* Correspondence: bozhiqian@263.net

${ }^{1}$ XUJ Group Corporation, Xuchang, China

Full list of author information is available at the end of the article
}

through a number of development stages, migrating from electro-mechanical to semiconductor, and subsequently to integrated circuit and microprocessor technologies. Today, microprocessor-based digital and numeric relays are replacing conventional relays in all areas of power system protection. However, many of the same relaying principles of protection are still playing a dominant role to date. In the late 1960s, the application of a centralized substation protection system based on a centralized computer system was proposed [1]. This constitutes an important milestone in the history of power system protection. The idea fits well with the concept of an overall integrated protection where the protection package would not only oversee individual units of a plant but also a section of the network. However, the idea has not been widely applied until recently, since there were no available computer hardware/software or communication technologies to support such an idea. Since then, relay technology has enjoyed successful developments based on the application of digital techniques. The introduction of microprocessors into protection in the 1980s generally 


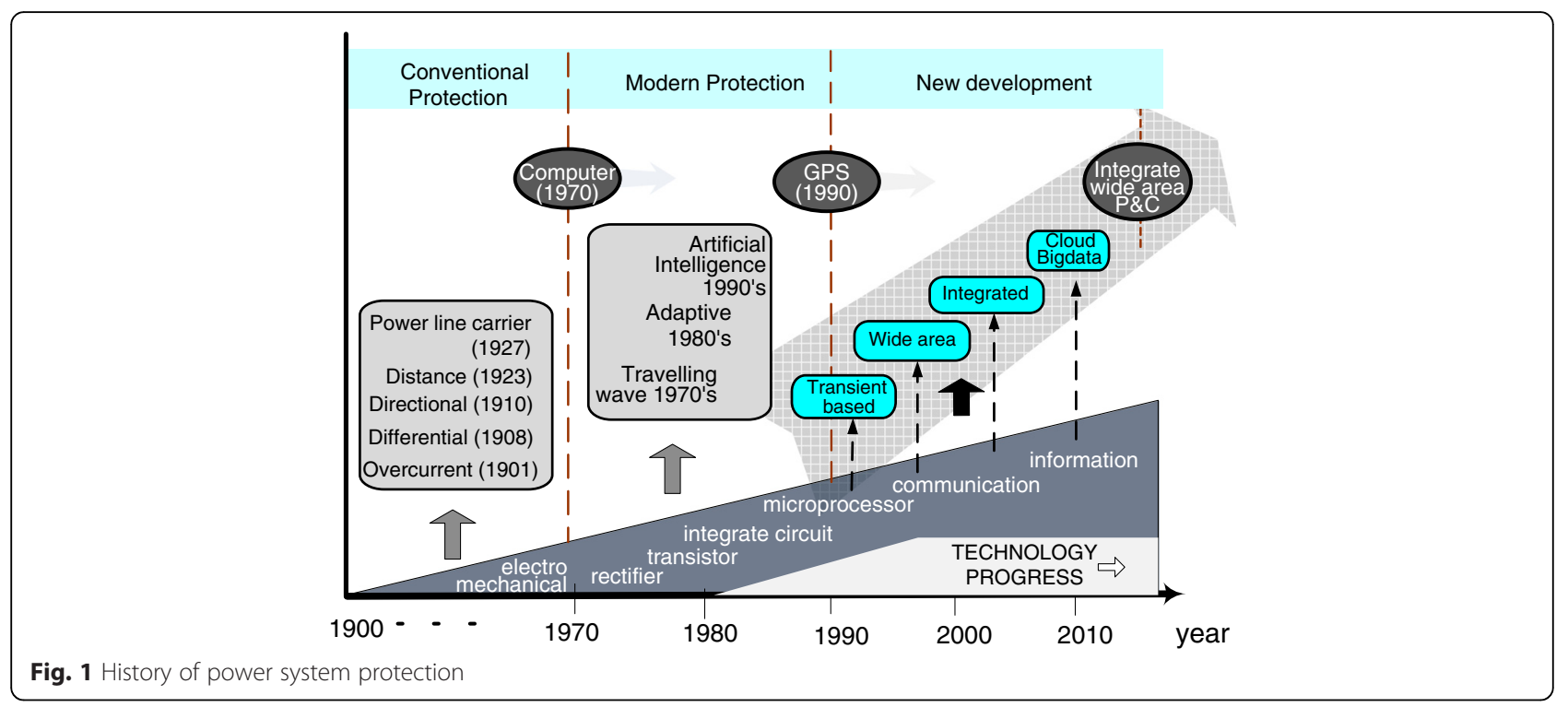

followed the conventional approach with the implementation of distributed processing platforms that concentrated on protecting individual units of the system. Limited integrated protection was provided in the form of back-up protection and thus remained a secondary function.

Developments in the 1980s and 1990s provided new means to advance power system protection, especially the 'Adaptive Protection' and the Artificial Intelligence (AI)-based protection techniques proposed in the 1980s and 1990s. The adaptive protection started with the application of Inverse Definite Minimum Time Overcurrent (IDMT) protection in the early time of protection history. The concept played an important role in the 1980s with the progress of computing technology and associated control theory. It can be defined as a new type of relay protection which can change the performance, characteristics or set value according to the operation mode and fault condition of the power system. The basic idea of adaptive relay protection is to protect the power system as much as possible to improve the performance of the protection. Adaptive relay protection has the advantages of improving the response of the system, enhancing the reliability and improving the economic benefits. It has a wide application prospect in the field of distance protection, transformer protection, generator protection, autoreclosure and so on. Research has discovered that, to achieve the protection of the system adaptive to the operation mode and fault status, more detailed system operation and fault information are required through communication network.

The 1990s witnessed the rapid development of electronic and computer technology, the artificial intelligence technology such as artificial neural networks, genetic algorithms, evolutionary algorithm, fuzzy logic and other research applications, which have been applied to the field of relay protection. For example, artificial neural network (ANN) is used to achieve fault type identification, fault distance measurement, direction protection, and so on. Artificial neural network has the characteristics of distributed storage, parallel processing, self-organization and self-learning. The application of artificial intelligence will improve the speed and accuracy of fault detection and analysis, which represents the future development of an intelligent diagnosis system.

As a result of these developments, the performance of the protection relays has been improved. However, these developments have concentrated on the improvement of conventional relaying techniques, and no significant new relaying principles have been derived from the application of the Adaptive and AI techniques. At the same time in the 1990s, with the continuous expansion of the power network, the demand for fast fault clearance to improve system stability encouraged research into nonpower system frequency fault detection techniques to increase the speed of the relay response. This led to the development of the so-called 'transient based protection' relays based on travelling wave and superimposed components, which utilizes the fault generated transients for transmission system protection. Studies have found that the fault generated high frequency transients can be detected and quantified, creating the possibility for developing new protection principles and techniques [2]. Considerable effort has now been devoted to research on high frequency transient detection. Another important milestone is the application of novel communication technique, the utilization of global positioning system (GPS) in power system protection as shown in Fig. 1. In this respect, a number of new techniques has been 
proposed. In particular, the new proposed protection relay principle is able to provide protection for wide area power network [3, 4]. Following the development, the concept of wide area protection focusing on control aspect has been presented [5].

In recent years, the dramatic growth in signal processing capability of relay platforms, and the availability of suitable communications schemes, have provided a new opportunity to revisit the concept of centralized protection. Research [6] on the concept of 'Integrated Protection' shows that information obtained from multiple power plants and components can be used to derive new protection principles and schemes, which could have significant advantages over the existing protection techniques based on the individual plant or component. In this respect, the substation area protection [7] has quickly become a practical development and application area. Furthermore, the development of information technology has resulted in the interests in utilizing cloud computing [8] and big data techniques, as shown in Fig. 1, to improve the performance of power system protection and control.

On the other front, the developments in the field of substation integrated automation technology provide the technical basis for optimizing the combination and system integration of monitoring, control, protection and measurement device and system. The implementation of relay protection and integrated automation reflects in the integration and resource sharing, remote control and information sharing. Taking the remote terminal unit and microcomputer protection device as the core, the control, signal, measurement, billing and other circuits are integrated into the computer system to replace the traditional control protection cabinet, which can reduce the area and equipment investment and improve the reliability of the secondary system. With the advanced computer and communication network, the relay protection device is actually a high performance and multifunction computer, which is an intelligent terminal of the whole power system computer network. It can obtain any information about the operation and fault of the power system from the network, and can transmit any information of the protected components to the network control center or any terminal. Therefore, each microcomputer protection device can complete not only the relay protection function, but also the measurement, control, data communication and other functions under normal operation condition, and can also realize the integration of protection, control, measurement and data communication.

\section{Recent development in power system protection and control}

With the fast progress in high-speed communication network and information technology, there were significant developments in power system protection, power system control and wide area control in recent years, particularly in the wide-area and integrated protection.

\section{Recent development Wide area protection}

In recent years, the fast development in communication technologies makes the wide-area information exchange possible. In this respect, the emergence of the wide area measurement system provides a new idea for the design of power system protection systems. The first wide-area protection principle is derived from the transient based protection in 1996 [2], in which GPS time synchronization played a major role in the design [3]. This was immediately followed by a summary paper in 1997, which systematically outlines the concept of the so-called "wide area protection" [4], focusing principally on the control aspect of the area. The wide area protection based on novel algorithms, which is derived from the measurements of multiple information points, is able to provide fast, reliable and accurate fault clearance, analyse the effects on the system stability based on the fault system analysis and take necessary control measures to perform the functions of relay protection, security, and stability control in order to prevent voltage collapse. Wide area relay protection has quickly become a hot research topic with many research results published particularly in recent years.

\section{Integrated protection}

With the development of digital technology, more and more protection functions for any given apparatus (line, transformer, generator, etc.) have been implemented within one protective device to achieve a certain degree of integration. For example, a numeric line protection relay may have distance or current differential function as the main protection, and directional and overcurrent functions as the backup protection. The recent developments in microprocessor and communication techniques provided new means to derive new protection principles and schemes based on the information obtained from multiple power plants and components, which could have significant advantages over the existing protection techniques based on the individual plant or component [5]. Unlike centralized protection (or substation area protection), the integrated protection does not simply centralize the relay hardware/software, but concentrates on the developments of new concepts and algorithms based on multiple points of measurements; via this means it is hoped that the performance of protection can be improved significantly. There was also research in the field of integrated wide area protection [9]. 


\section{Wide area control}

The increased deployment of wide-area measurements will significantly enhance the power system wide-area power system operation and control. They provide voltage and current phasor information, synchronized with high precision to a common time reference provided by GPS. Therefore, a wide range of power system monitoring and control applications can be implemented in the system for improving system awareness and reliability, which includes enhanced state estimation based on mixed Remote Terminal Unit (RTU) and Phasor Measurement Unit (PMU) measurements [10], dynamic model online estimation and validation [11], real-time congestion management, real-time stability estimation [12], detection and damping of inter-area oscillations [13]. However, the most important and challengeable applications are the implementations of wide area stability real-time detection and control to prevent blackouts [14]. There was also research in the integrated protection and control [15].

\section{New concept and development}

Based on the developments mentioned above, a new concept of the integrated wide area protection and control (IWAPC) has been proposed recently. The main focus of the concept $[16,17]$ is the integration between the protection and control, particularly at the wide-area or regional level, aimed to provide a number of benefits to the future protection and control system, e.g., the potential to merge the three lines of defence system and on-line self-healing decision making, in order to prevent cascading tripping of large area power network. The concept of integrated wide area protection and control is introduced, in which a three-level hierarchically coordinated system, supported by the specially designed realtime synchronised wide-area communication network, provides the protection and control for wide area or regional power substations/plants and their associated power network. The key element in the system is the integrated wide area protection and control information platform, which receives real-time synchronised data from the communication network to support the integration of protection and control at the wide area/regional level.

The information platform also supports the application of a cloud computing system, which is specially designed to implement a number of secondary functions for substations and power networks. In addition to the basic functions of relay protection, the platform should have a large capacity of fault information and data storage, fast data processing functions, powerful communication functions, and other protection, control devices and scheduling network to share the whole system data, information and network resources, and can also carry out remote monitoring with the computer monitoring system of substation communication. With the proposed platform, the architecture of future substation equipment may be reshaped to provide a flexible framework for building an interactive grid and subsequently improve the reliability and security of power grids.

\section{Integrated wide area/regional protection and control \\ Architecture of integrated wide area protection and control}

The proposed integrated wide area or regional protection and control system (IWAPC) is illustrated in Fig. 2. There have been fast developments in both power transmission and distribution networks, e.g., the series compensation in $\mathrm{AC}$ lines and high-voltage $\mathrm{DC}$ lines in transmission systems, distributed generation and energy storage in distribution systems, etc. These new developments result in far more complicated characteristics than that of conventional systems. Consequently, the existing protection and control system will no longer be effective to cope with the new systems, and this has led to the proposed IWAPC system. As is shown, the IWAPC system consists of different equipment at different layers: from bottom to top, there is the integrated multiple-function intelligent equipment at the local level; the substation communication network and the integrated substation protection and control at the substation level; the wide area communication network, the integrated wide area information platform and the integrated wide area (regional) protection and control at wide area level. The key parts of the system are the high-speed wide area communication network and the real-time synchronisation information platform.

The IWAPC is further extended to dispatching in order to achieve the integration of dispatching automation, protection and control of power grid, and according to the three-level dispatching (country, province, regional) architecture to implement the functions of regional protection, control and dispatching managements.

\section{Multiple functions intelligent equipment at the local level}

As shown in Fig. 2, the Intelligent equipment at local level is an integrated multiple function secondary equipment in the substation, which mainly consists of the $\mathrm{MU}$, intelligent terminal, metrology measurement, PMU \& local protection. The equipment is responsible for sampling all real-time data and sending information to the integrated substation $P \& C$ and wide area $P \& C$. It also receives and carries out the control commands from the integrated substation $\mathrm{P} \& \mathrm{C}$ and the IWAPC. The equipment can be integrated into primary power apparatuses and achieve local protection for $90 \%$ of its associated line sections. It has a redundant configuration to 


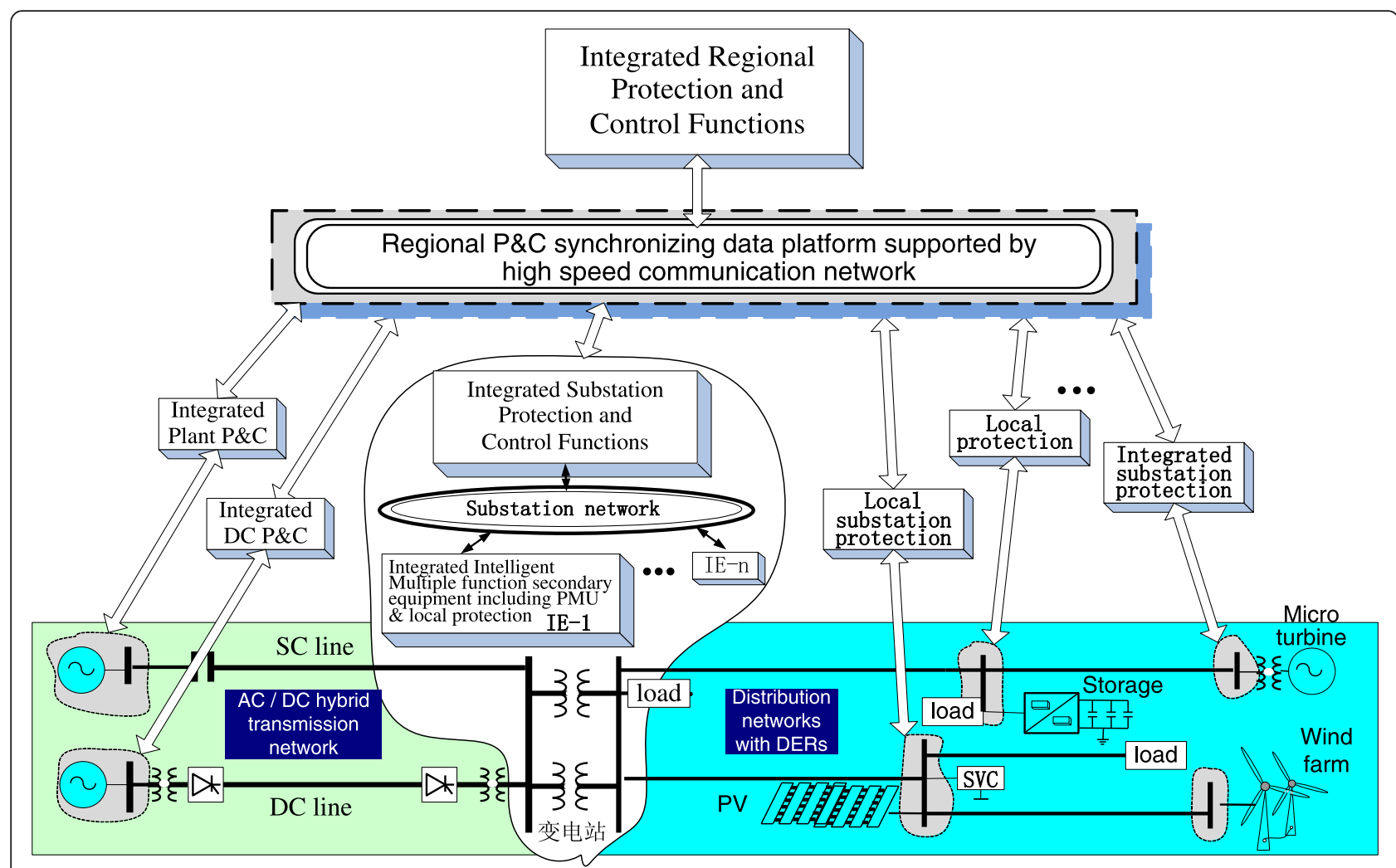

Fig. 2 Integrated wide area protection and control

ensure reliability, together with other integrated functions such as fault recorder, data storage and network analysis, etc.

\section{Integrated substation protection and control at the substation/plant level}

The substation $P \& C$ integrates functions of line, bus, transformer protections, switch failure; autoreclosure, automatic bus transfer, UFLS, UVLS, overload intertripping and substation control function, etc. It utilizes information from the entire substation to achieve substation backup protection and safety automatic control, etc. The CBs are used as units to configure the adaptive backup protection, and current differential protection is used to replace the stage overcurrent protection, breaker failure protection and dead zone protection in the conventional protection system.

\section{Integrated wide area/regional protection and control}

The IWAPC specially designed for the protection and control of power network is able to offer fast protection. In addition, they both integrate functions of automatic UFLS and UVLS, voltage and frequency control, oscillation detection and out-of-step separation, etc. In addition, the IWAPC also incorporates the function of transmission cross-section safety $\mathrm{P} \& \mathrm{C}$.
Unlike conventional protection and control, which are separated in both design and operation, the IWAPC integrates protection and control into one optimal combined system, which effectively coordinates the wide area (regional) protection and control, in order to achieve significant improvements in the protection and control of power systems.

\section{Synchronised high speed communication network}

One of the most important elements of the IWAPC system is the fast communication network. In this respect, the latest development in communication network, the Packet Transport Network (PTN) may be a better choice to implement such a task. The present power communication network is mainly used in multi-service transport platform based on the Synchronous Digital Hierarchy (SDH). Its advantages lie in its high efficiency for carrying TDM services, low latency, high reliability, with end management capabilities. However, with the new trends in smart grid development, SDH technology gradually revealed its limitations, such as low bearing efficiency and poor flexibility for data services. In contrast, PTN can realise statistical multiplexing and efficient transfer of packet service by using packet-switched core, which can overcome the weaknesses of SDH rigid bandwidth. In addition, it can provide good quality of service, 
operation, administration and maintenance. Self-healing fibre optical network is employed to connect a number of substations in the region, to ensure full sharing of dynamic and transient information for all electrical measurements, breaker status and protection operations; using high reliability IEEE-1588 technology to ensure the synchronization timing of the sharing data, to prove the data for the integrated wide area protection and control. However, SDH is still an option for the task since it has been widely applied in power network.

\section{Synchronized information platform}

Substation is installed with a wide range of electrical equipment with complex designs and is difficult to maintain. With the continuing improvement in power system automation and the intelligence level, the system network has been expanding, along with the huge amount of information in protection and control. As each piece of information is collected and stored by different devices in each separate system, the interoperability of the internal power system data between systems is poor, whereas complex communication protocols tend to create information islands. Consequently, the measurement data and protection control mechanism cannot be shared, which restricts the information integration. The protection and control of smart grid requires dealing with the new situation demands of the application, in order to improve further the information platform capabilities for the future development of key technologies, and to make the information platform system more open.

The real-time synchronized information platform accurately collects wide area information and conducts data mining to investigate the logic relation between the real-time information to increase the sensitivity, reliability and fault tolerance capability. The data received from the platform includes static, dynamic, transient measurements and states of circuit breakers, etc. Valuable information is extracted from the data and allocated to various specially-designed computation algorithms in the platform to perform advanced functions of protection and control for the power network. In the platform, sets of data need to be transferred and their transferring speed depends on the application, e.g., slow speed for contingency analysis, near real time speed for monitoring, real time speed for control, and high speed for wide area protection,; in particular, time synchronization. The information can also include other types of data, such as the oil and ambient temperature of the transformer, wind speed and direction, sun intensity, etc. On the other hand, the information is stored in a hierarchical manner instead of a centralized one, which comprises the hierarchical protection and control system. Equipped with the latest high-speed synchronised communication technology, integrated with the advanced protection techniques and the latest developments in control system, the system offers not only fast protection, but also complete control of entire power network.

The advanced computing technology is introduced to establish a synchronized information platform for wide area protection and control, to build a panoramic operation and maintenance data collection network, providing a standardized interface to the terminal device, to form a resource sharing, flexible and interactive, open and ordered information platform. In summary, advanced computing technologies are used to build a distributed collaborative intelligent information platform, simplifying terminal data collection equipment, and breaking the barriers between protection and control systems at different substations through the specially designed synchronized information platform.

\section{Wide area power cloud}

Based on the information platform mentioned above, a distributed cloud system is designed to implement functions at substation and regional levels, such as wide area fault location, fault line selection, power quality monitoring, protection settings, etc. The extended functions also include the equipment monitoring, life cycle and operation management, as shown in Fig. 3.

Currently, many kinds of secondary equipments achieving different functions are installed in each substation, and an increasing number of distributed energy resources of small capacity added to the system greatly increase the number of equipments. To implement these equipments, complex functions in a specially developed distributed "cloud" system will greatly reduce the equipment investment. The cloud at substation level receives the data from process level, and the regional cloud receives the data from the information platform, which includes static, dynamic, transient measurements and states of circuit breakers, extracting valuable information and allocating them to various specially-designed computation algorithms in the platform to perform advanced functions in order to identify the faulted line, the accurate fault location and the contents of harmonics, etc.

The cloud computing platform can make full use of "processing ability of cloud" to reduce the burden of terminal secondary equipment. Based on big data technique, the computing clouds enjoy strong processing power based on demand. There is no need for endless upgrades to improve the processing capacity of the equipment, and there is also no need to update the software to achieve a variety of task processing. There are many more advantages which can be derived from the cloud system, such as the wide area information sharing, standardization of software and algorithm, reduction of 


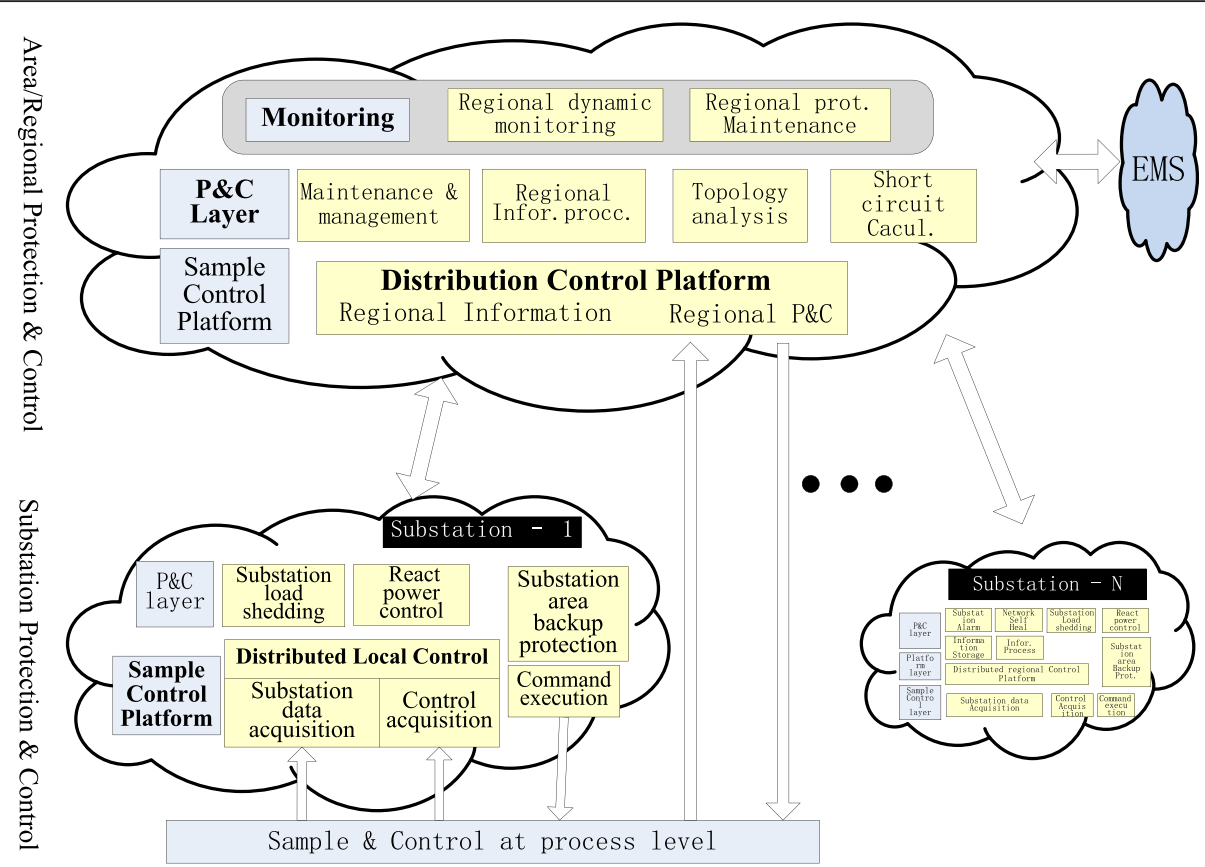

Fig. 3 Structure of distributed power cloud

equipment investment, substation area occupation and work load for operation and maintenance.

\section{Conclusions}

This paper presents an integrated wide area protection and control system based on a hierarchical structure, which integrates protection and control at local, substation and regional levels. Covering both transmission and distribution networks, the system is supported by the proposed high-speed synchronised communication network and the real-time protection and control information platform. The system, which integrates the advanced protection techniques and the latest developments in control system, offers not only fast protection, but also complete control of the entire power network. It offers a potential for the merger of the three lines of defence into a unified system to ensure more effectively the reliable and safe operation of power grid. Based on the system information platform, a distributed power cloud system is also designed to support many advanced applications for the integrated wide area protection and control.

With the continuous advances in measurement, communication and information technologies, the system presents a bright future for practical application. Overall improved performance of protection and control can be expected from the proposed system. However, for the system to become useful in power system application, it is equally important that its practical implementation be readily manageable, user-friendly and cost-effective. The authors hold that, to achieve these goals, the proposed integrated protection and control at wide area level offers an appealing way forward.

\section{Authors' contributions}

ZB investigated the framework of integrated wide area protection and control, and drafted the manuscript. XL summarized the history of power system protection. QW summarized recent development of power system protection. YY summarized the wide area power cloud. FZ participated in typesetting and revision of the manuscript. All authors read and approved the final manuscript.

\section{Competing interests}

The authors declare that they have no competing interests.

\section{About the authors}

Zhiqian Bo received his BSc degree from the Northeastern University, China in 1982 and PhD degree from The Queen's University of Belfast, UK in 1988 respectively. From 1989 to 1997, he was with the Power and Energy Group, University of Bath, UK. He has been with ALSTOM Grid Automation and responsible for new technology development and international research collaboration from 1998 to 2012. Recently he joined the XUJI Group of the State Grid Corporation of China (SGCC) as the Chief Expert on smart grid. Xiangning Lin (SM'08) received the M.S. and Ph.D. degree in school of electrical and electronic engineering of Huazhong University of Science and Technology (HUST), Wuhan, China. Currently, he is a professor at HUST. His research interests are modern signal processing and its application in power systems as well as power system protective relaying and control. Qingping Wang was born in Hebei Province, China, in 1975. He received his Ph.D in 2002 from the Department of Electrical Engineering, Tianjin University, China. At present, he has been employed as CTO of XJ Group Corporate Research. His research interests include protection, substation \& distribution automation, power system simulation and micro-grid. He is author or co-author of more than 40 journal papers and owner of more than 30 invention patents. Yonghui Yi, born in 1969, PhD in electrical engineering. Currently he works as the Managing Director of Protection and Automation Company of XUJ Group, His main research areas are smart grid and power system protection. Fengquan Zhou, born in 1969, is the director of R\&D Center, XJ Group, obtained his first Degree and graduate Degree from Tsinghua University, 
finished his PHD research in McGill University in Canada. His research areas include energy efficiency, power system automation protection, distributed generation, renewable energy, smart grid and other fields.

\section{Author details}

${ }^{1} X U J I$ Group Corporation, Xuchang, China. ${ }^{2}$ Huazhong University of Science and Technology, Wuhan, China.

Received: 9 May 2016 Accepted: 9 May 2016

Published online: 19 June 2016

\section{References}

1. Rockefeller, G. D. (1969). Fault protection with digital computer. IEEE Transactions on Power Apparatus and Systems, 88(4), 438-461.

2. Bo, Z.Q., Jiang, F., Chen, Z., et al. (2000). Transient based protection for power transmission systems. In: IEEE PES Winter Meeting. Singapore.

3. Bo, Z.Q., Jayasinge, J.A.S.B., Aggarwal, R.K., et al. (1996). A new scheme for monitoring and protection of power transmission system based on global positioning system. In: Technol. Educ. Inst. Iraklio The 31st University Power Engineering Conference (pp. 21-24). Crete.

4. Bo, Z. Q., Weller, G., \& Lomas, T. (2000). Positional protection of transmission system using global positioning system. IEEE Transactions on Power Delivery, 15(4), 1163-1168.

5. Ingelsson, B., Lindstrom, P. O., Karlsson, D., et al. (1997). Wide-area protection against voltage collapse. IEEE Computer Applications in Power, 10(4), 30-35.

6. Bo, Z. Q., He, J. H., \& Dong, X. Z. (2005). Integrated protection of power network. Relay, 33(14), 33-41.

7. Gao, H. L., Liu, Y. Q., Su, J. J., et al. (2012). New type of substation-area backup protection for intelligent substation. In 2012 China Smart Grid Seminar.

8. Bo, Z.Q., Wang, L., Zhou, F.Q., et al. (2014). Substation cloud computing for secondary auxiliary equipment.In: IEEE Powercon2014. Chengdu.

9. Bo, Z. Q., Zhang, B. H., Dong, X. Z., He, J. H., et al. (2013). The development of protection intellectuation and smart relay network. Power System Protection and Control, 41(2), 1-12.

10. Guo, Y., Wu, W. C., Zhang, B. M., et al. (2015). A distributed state estimation method for power systems incorporated with linear and nonlinear models. International Journal of Electrical Power \& Energy Systems, 64, 608-616.

11. Chen, R.Z., Wu, W.C., Sun, H.B., Zhang, \& B.M. (2013). A two-level online parameter identification approach. In: IEEE Power \& Energy Society General Meeting. Vancouver.

12. Liu, M.C., Zhang, B.M., Yao, L.Z., et al. (2008). PMU based voltage stability analysis for the transmission corridor. In: IEEE The 3rd Int. Conf. on Electric Utility Deregulation and Restructing and Power Technologies. Nanjing.

13. He, J. B., Lu, C., Wu, X. C., et al. (2007). Design and experiment of heuristic adaptive HVDC supplementary damping controller based on online Prony analysis. In Proc. IEEE Power Engineering Society General Meeting.

14. Wu, W.C., Zhang, B.M., Sun, H.B., et al. (2010). Development and application of on-line dynamic security early warning and preventive control system in China. In: IEEE Power \& Energy Society General Meeting. Mineapolis.

15. Wang, B., Dong, X. Z., Xu, F., Cao, R. B., Liu, K., \& Bo, Z. Q. (2011). Analysis of data sharing for protection and control system in smart distribution substation". In Proceedings of the CSEE (Vol. 31).

16. Bo, Z.Q., Ge, S.M., Wang, Q.P., Wang, L., Zhou, F.Q., Liu, X., \& Fan, Z.F. (2015). Integrated wide area protection and control systems based on advanced communication network. In: ATLANTIS PRESS 2015 International Conference on Mechanical Engineering, Materials and Energy. Tianjin.

17. Bo, Z.Q., Wang, Q.P., Wang, L., Zhou, F.Q., Ge, S.M., \& Zhang, B.M. "Architecture design for integrated wide area protection and control systems", APPEEC, April 14 2015, The $7^{\text {th }}$ Asia-Pacific Power and Energy Engineering Conference (APPEEC 2015).

\section{Submit your manuscript to a SpringerOpen ${ }^{\odot}$ journal and benefit from:}

- Convenient online submission

- Rigorous peer review

- Immediate publication on acceptance

- Open access: articles freely available online

- High visibility within the field

- Retaining the copyright to your article

Submit your next manuscript at $\boldsymbol{s p r i n g e r o p e n . c o m ~}$ 